\title{
Validated high-performance thin-layer chromatographic analysis of curcumin in the methanolic fraction of Curcuma longa $\mathrm{L}$. rhizomes
}

\author{
Poonam Kushwaha ${ }^{1 *}$, Babita Shukla1', Jyotsana Dwivedi² and Sumedha Saxena ${ }^{1}$
}

\begin{abstract}
Background: In the present study, an HPTLC (high-performance thin-layer chromatography) method was developed for the quantitative determination and validation of the curcumin in the methanolic fraction of Curcuma longa L. For achieving good separation of curcumin, the mobile phase of chloroform:methanol (97:3) was used. The densitometric analysis of curcumin was performed at $420 \mathrm{~nm}$ in reflection/absorption mode.

Results: Linearity of the method was obtained in the range of 100-600 ng per spot. During analysis, the methanolic fraction of the $C$. longa showed the presence of a quantifiable amount of curcumin. The content of curcumin was found to be $1.5 \%$ (per dry weight).

Conclusions: The method is specific, simple, precise, and accurate. The obtained data can have used for the routine analysis of the reported biomarkers in crude drugs and extracts. The quantification and the method validation of curcumin have not yet been reported in C. longa which can be utilized for the proper standardization of the plant.
\end{abstract}

Keywords: Curcumin, Curcuma longa, High-performance thin-layer chromatography, Validation

\section{Background}

Curcuma longa L. (Zingiberaceae) is a rhizomatous perennial herb. It is a medicinally important plant commercially known as Haldi, Turmeric, or Indian saffron. It has a long history of traditional uses ranging from folk medicine to various culinary preparations. The paste of C. longa rhizome is commonly used traditionally in the treatment of asthma, leucoderma, tumor, piles [1]. In the Indian system of medicine, it is well reported as an anti-inflammatory and skin protective agent [2]. C. longa is the potential source of many secondary metabolites namely curcuminoids, curcumin, demethoxycurcumin, bisdemethoxycurcumin, turmerone, atlantone, and

\footnotetext{
*Correspondence: Poonam.kushwaha083@gmail.com

1 Faculty of Pharmacy, Integral University, Lucknow, India

Full list of author information is available at the end of the article
}

zingiberone, sesquiterpenoids, sugars, resins, proteins, vitamins, and minerals.

Curcumin is the major active constituent of $C$. longa. Structurally, Curcumin is 1,7-bis(4-hydroxy3-methoxyphenyl)-1,6-heptadiene-3,5-dione (Fig. 1). It is a natural polyphenol procured from the rhizomes of C. longa [3]. It is a bright yellow-orange colored powder. Curcumin exhibits various pharmacological activities including antioxidant, anti-inflammatory, anticarcinogenic, hypocholesterolemic, wound healing, antispasmodic, anticoagulant, antitumor, and hepatoprotective activities [2]. Curcumin is included in various herbal remedies to treat skin inflammation and its infection. It is therapeutically used for the treatment of amenorrhea, dislocation of joints, diarrhea, diabetes, liver disorder, bronchitis, ringworm infection, toothache, anemia, and weakness of eyesight $[1,4,5]$. 


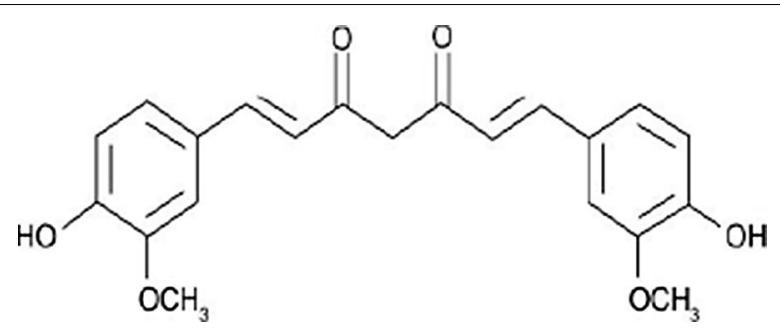

Fig. 1 Structure of curcumin

The variation in the content of phytoconstituents in herbal extract presents a major challenge to control the quality of herbal products. To assess the safety and efficacy of plant material, qualitative and quantitative analyses are useful techniques which mostly related to the content of marker compounds present in the plant [6, 7]. Curcumin, the principal active constituent largely responsible for the therapeutic efficacy of C. longa, is recognized as the marker compound [2].

In recent years, the advancement of chromatographic and spectral fingerprints plays an important role in the quality control of herbal medicines [5]. Chromatographic fingerprint investigation has been demonstrated to be a realistic and practical method for quality assessment and authentication of various herbal medicines. This method can be used to identify the presence or absence of markers as well as the ratio of all detectable compounds [6]. Although several methods for the quantification of curcumin were reported by various researchers including supramolecular solvent-based liquid-liquid microextraction (SMS-LLME) [7], isocratic high-performance liquid chromatography (HPLC) [8, 9], ultra-performance liquid chromatography-mass spectrometry (UPLCMS) [10], and reversed-phase liquid chromatography (RPLC) [11]. However, HPTLC has become a routine analytical technique due to its advantages $[5,12,13]$. The TLC chromatogram pattern comparison seems to be promising for fingerprinting the active compounds in plant extracts. HPTLC has been known as the fast tool for the detection of compounds [6]. Analytical quantification of chemical markers through HPTLC has the advantage of combining chromatographic separation on a silica layer, along with in situ densitometric quantification of the separated compounds [13]. This results in an efficient, quick, accurate, and relatively inexpensive method for the quantification of separated phytoconstituents [14], thus eliminating the possible interference given by other structurally related compounds. HPTLC method provides many other benefits such as rapid analysis time, i.e., many samples can be analyzed simultaneously, low solvent usage on a persample basis, low operating cost, high sample throughput, and the need for minimum sample clean-up. It can detect more compounds than HPLC, although the resolution is poorer $[5,6,13]$. Additionally, the compounds having no UV absorption can be detected by reagent spraying. Utilizing a data analysis system and optimized experimental conditions, HPTLC is also feasible for the development of chromatographic fingerprint methods to determine and identify complex herbal extracts just like HPLC and GC. The compounds which cannot be eluted still can be detected. Furthermore, the colorful picture, like the HPTLC image, provides extra-intuitive parameters of visible color and/or fluorescence. Moreover, the colorful pattern and quantification at the micron and nanogram levels help to differentiate various samples on the same plate [12-14].

Comparing to the GC and HPLC, the HPTLC method has few restrictions, such as a low plate efficiency and narrow developing distance. Despite that, it remains a valuable tool for quality assessment of natural products due to its ease, low cost, and few requirements, and it has been profitably utilized to develop a chromatographic fingerprint for various natural products, herbal drugs, and commercial herbal formulations [5, 6]. However, with the advancement in analytical techniques, a smartphone-enabled alternative of TLC densitometric scanning was developed which is economic, precise, and accurate to serve the purpose. Such techniques are especially effective in low-income countries and the methods developed with traditional HPTLC can easily be reciprocated to Smartphone-based image analysis $[15,16]$. But this method requires technical experience.

Looking into the benefits, in the present work, a densitometric HPTLC method has been developed and validated according to International Conference on Harmonization ( $\mathrm{ICH})$ guidelines for the quantitation of curcumin from methanolic extract C. longa.

\section{Methods}

\section{Chemicals and reagents}

Curcumin was purchased from Sigma-Aldrich (Steinheim, Germany). HPTLC plates $(20 \mathrm{~cm} \times 10 \mathrm{~cm}$, precoated silica gel aluminum plates 60 F254 $(0.25 \mathrm{~mm})$ were procured from E. Merck (Darmstadt, Germany). Methanol and chloroform were obtained from Merk (Mumbai, India). All other ingredients were of analytical grade. Double-distilled water (DDW) was used for all experiments.

\section{Plant material}

The plant materials, i.e., rhizome parts of $C$. longa, were collected from Lucknow, India (Fig. 2). 


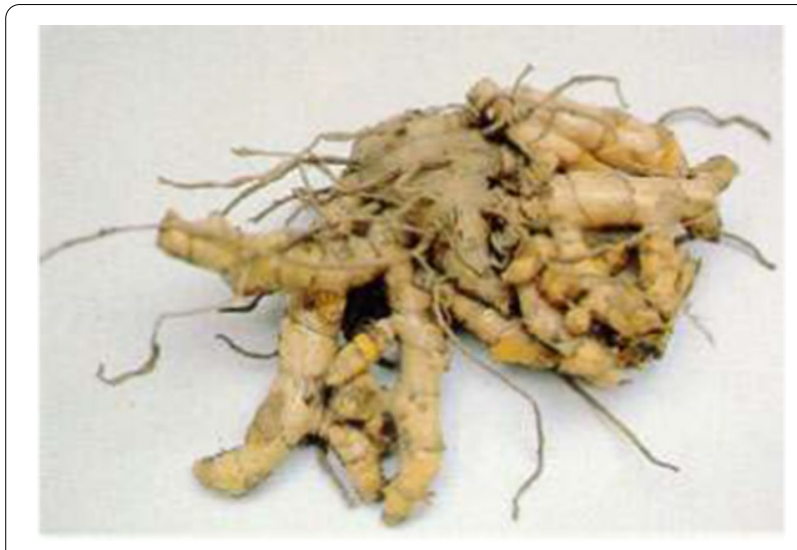

Fig. 2 Fresh rhizomes of Curcuma longa $\mathrm{L}$

\section{Standard preparation}

Stock solutions of curcumin were prepared by dissolving $0.1 \mathrm{mg} \mathrm{mL}^{-1}$ of curcumin in the methanol.

\section{Sample preparation}

The rhizome parts of $C$. longa were collected, thoroughly washed with the water to remove all the debris. The plant materials were shade-dried and coarsely powdered using an electric grinder at 60 mesh size. Powdered materials were defatted using $98 \%$ petroleum ether to remove the fatty materials/impurities. Defatted materials were extracted by the hot continuous extraction (soxhlation) method. Soxhlation was done for $9 \mathrm{~h}$ using $250 \mathrm{~mL}$ chloroform, followed by the methanol. The obtained methanolic fraction was passed through the filter paper (Whatman No.1). The filtrate was concentrated under vacuum in the rotary evaporator at $40{ }^{\circ} \mathrm{C}$ and stored at $4{ }^{\circ} \mathrm{C}$ for further use. The dried extracts were dissolved in $98 \%$ methanol to obtain the stock solution of $10 \mathrm{mg} / \mathrm{mL}$ for the application of spots on HPTLC plates.

\section{Development of HPTLC fingerprinting of curcumin Instrumentation and chromatographic conditions}

The instruments and chromatographic conditions used are as follows:

- HPTLC chamber: Glass twin-trough chamber $(20 \times$ $10 \times 4 \mathrm{~cm}$ ) CAMAG.

- Densitometer: HPTLC Scanner 3 linked to win CATS software V. 4.06 CAMAG.

- Spotting device: Linomat V automatic sample applicator CAMAG (Muttenz, Switzerland).

- HPTLC plates: $20 \times 10 \mathrm{~cm}, 0.2 \mathrm{~mm}$ thickness precoated with the silica gel $60 \mathrm{~F}_{254}$ (E-Merck).

- Band size: $6 \mathrm{~mm}$;
- Slit dimension: $5.00 \times 0.45 \mathrm{~mm}$.

- Syringe: $100 \mu \mathrm{L}$ Hamilton (Bonaduz, Switzerland).

- Scanning speed: $10 \mathrm{~mm} \mathrm{~s}^{-1}$

- Radiation source: deuterium lamp.

- Sample volume: $10 \mu \mathrm{L}$

- Experimental conditions: temperature, $25 \pm 2{ }^{\circ} \mathrm{C}$; relative humidity, $40 \%$.

- Solvent system: chloroform: methanol $(97: 3 \mathrm{~V} / \mathrm{V})$.

- Detection wavelength: $420 \mathrm{~nm}$.

\section{Calibration curve of curcumin}

A stock solution of curcumin $\left(100 \mu \mathrm{g} \mathrm{mL}{ }^{-1}\right)$ was prepared in the HPLC grade methanol. The stock solution in different volumes was spotted on the HPTLC plate to achieve the concentrations of 100-600 ng per band of curcumin. Quantification was carried out by plotting data of peak areas against corresponding concentrations of the standard marker using regression analysis.

\section{Method validation}

Method validation was carried out to assess the suitability of the analytical method employed for its intended use. The developed method was validated in terms of linearity, precision, accuracy, limits of detection (LOD), limits of quantification (LOQ), and recovery as per the ICH guidelines [17, 18].

\section{Specificity}

The specificity of the method was determined by analyzing the band of curcumin and extract. The band of curcumin in samples was confirmed by the comparisons of the retention factor $\left(R_{f}\right)$ values and spectra of the band with standards. The peak purity of curcumin was confirmed by the comparing of the spectrum at peak initiation $(S)$, peak apex $(M)$, and peak-end $(E)$ positions of bands.

\section{Robustness of the method}

The robustness of the method was analyzed after introducing small modifications in the chromatographic conditions such as duration of mobile phase saturation, mobile phase composition, mobile phase volume, and activation of prewashed HPTLC plates with the methanol. Analysis was performed in triplicate at the concentration level of $200 \mathrm{ng}$ per band for curcumin. The relative standard deviation (RSD) and standard deviation (SD) of peak areas were calculated to assess the robustness of the method.

Limits of detection (LOD) and limits of quantification (LOQ) For the determination of the limit of detection (LOD) and limit of quantification (LOQ), different dilutions of 
known concentration of curcumin were spotted along with blank methanol for the six times and signal-to-noise $(\mathrm{S} / \mathrm{N})$ ratio was determined. A standard sample at the concentration level of $200 \mathrm{ng}$ per band was prepared and analyzed on day 0 and after $6,12,24,48$, and $72 \mathrm{~h}$. LOD was estimated at an S/N of 3:1 and LOQ at 10:1. Results of the analysis were treated for \% RSD to assess the ruggedness of the method.

\section{Accuracy}

The accuracy of the method was assessed by measuring the recovery of the curcumin at different concentration levels in the extract. Experimentally, the pre-analyzed samples were spiked at three different levels, i.e., 50, 100, and $150 \%$ of the standard curcumin. The experiment was conducted six times and \% recovery and average \% recovery of curcumin was calculated.

\section{Repeatability}

Repeatability of the sample application and peak area measurement was conducted at concentration levels of 200, 400, and $600 \mathrm{ng}$ per band of curcumin using nine determinants (3 concentrations $/ 3$ replicates). Repeatability reflects the reproducibility of the method for the quantification of the targeted analyte.

\section{Precision}

Intra- and inter-day precision analyses were performed at three different concentration levels viz 200, 400, and $600 \mathrm{ng}$ per band. For intra-day precision samples were scanned three times a day. For inter-day precision analysis, samples were analyzed for five consecutive days.
Results of the precision analysis were expressed in terms of mean relative standard deviation (RSD) (\%).

\section{Results}

\section{High-performance thin-layer chromatographic method} optimization

To provide an accurate, precise, and reproducible method for the determination of curcumin, HPTLC conditions were optimized in terms of selection of mobile phase, absorption maxima, and slit dimensions. Solvent systems in different combinations were tried. The absorption spectrum of curcumin was obtained at $420 \mathrm{~nm}$ (Fig. 3).

\section{High-performance thin-layer chromatographic method validation}

HPTLC profile (Fig. 4) with chromatogram was obtained for the standard compound, and the plant extract and targeted compound from the targeted plant

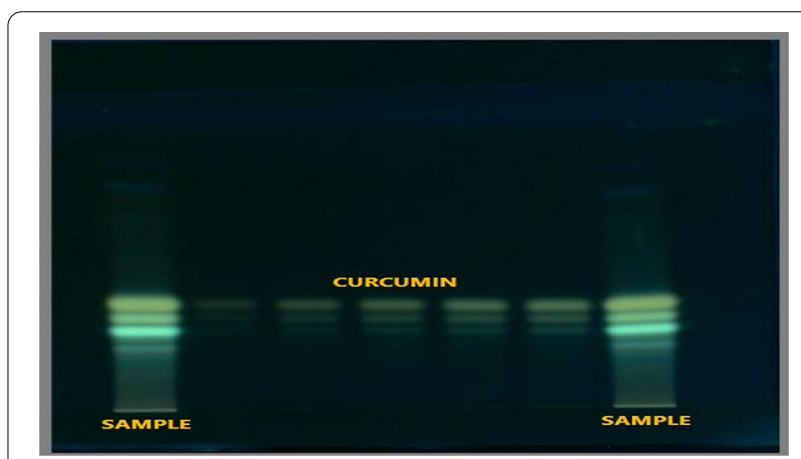

Fig. 4 HPTLC profiling of C. longa methanolic fraction with curcumin

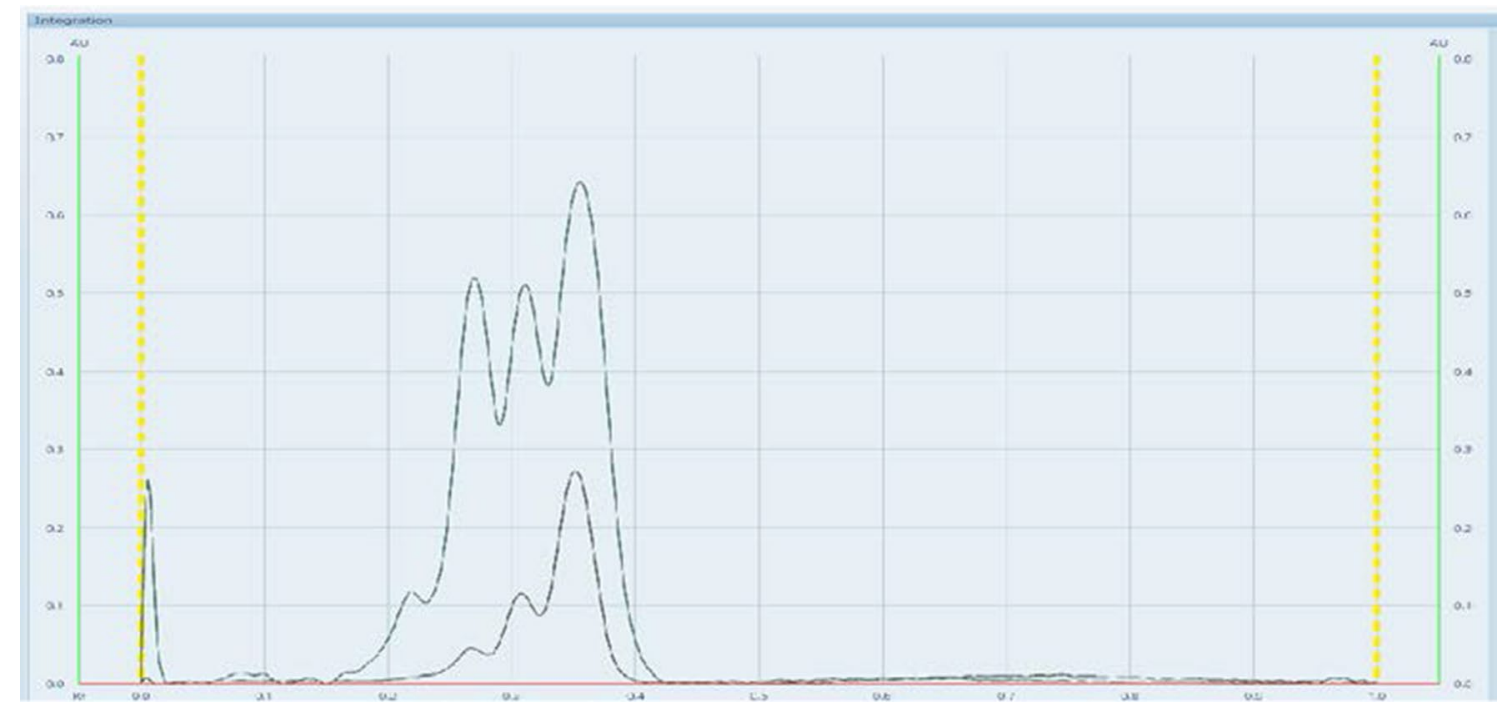

Fig. 3 UV spectrum of curcumin 
Table 1 Statistical parameter for linearity validation of curcumin

\begin{tabular}{ll}
\hline Parameters & Curcumin \\
\hline Linearity range $(\mathrm{ng} / \mathrm{spot})$ & $100-600$ \\
Retention factor $\left(R_{f}\right)$ & $0.37 \pm 0.04$ \\
Correlation coefficient $\left(r^{2}\right)$ & 0.9998 \\
Slope & 17.2 \\
Intercept & 907.9 \\
LOD $(\mathrm{ng} / \mathrm{spot})$ & 35 \\
LOQ $(\mathrm{ng} / \mathrm{spot})$ & 100 \\
Scanning $(\mathrm{nm})$ & 420 \\
Regression & $Y=17.2 x+907.9$ \\
\hline
\end{tabular}

was identified by the $R_{f}$ and the peak purity. The linearity of the method for the quantification of curcumin was obtained at the concentration of 100-600 ng/spot with a statistically, acceptable regression coefficient $\left(r^{2}\right)$ of 0.9998 . Other statistical parameters of the regression are found to be within the limit of acceptance as summarized in Table 1, which confirms the linearity of the developed method.

Specificity of the developed method indicates the clear and complete separation of marker(s), i.e., curcumin peak (Fig. 5). The \% RSD values of 0.31 in the intra-day analysis and 1.48 in inter-day analysis (Table 2). The recovery of curcumin in C. longa extract was found in the range of $97.48 \%$ to $98.15 \%$ (Table 3). The \% RSD value was found to be less than $5 \%$ which confirms the robustness of the method. The concentration of curcumin in the methanolic fraction of C. longa is represented in Table 4.
Table 2 Intra -day and Inter-day precision

\begin{tabular}{|c|c|c|c|c|c|}
\hline \multirow{2}{*}{$\begin{array}{l}\text { Standard } \\
\text { marker }\end{array}$} & \multirow{2}{*}{$\begin{array}{l}\text { Concentration } \\
\text { (ng) }\end{array}$} & \multicolumn{2}{|c|}{ Intra-day } & \multicolumn{2}{|c|}{ Inter-day } \\
\hline & & $\%$ RSD & Mean RSD & $\%$ RSD & Mean RSD \\
\hline \multirow[t]{3}{*}{ Curcumin } & 200 & 0.29 & & 1.85 & \\
\hline & 400 & 0.19 & 0.31 & 1.09 & 1.48 \\
\hline & 600 & 0.45 & & 1.5 & \\
\hline
\end{tabular}

Table 3 Recovery analysis of curcumin

\begin{tabular}{lllll}
\hline Standard & $\begin{array}{l}\text { Amount } \\
\text { added (\%) }\end{array}$ & $\begin{array}{l}\text { Amount recovered } \\
\text { (\%) mean }\end{array}$ & SD & RSD \\
\hline Curcumin & 50 & 97.48 & 1.65 & 1.69 \\
& 100 & 99.45 & 1.12 & 1.13 \\
& 150 & 98.15 & 1.67 & 1.68 \\
\hline
\end{tabular}

\section{Discussion}

Curcumin is an important secondary metabolite obtained from C. longa [2]. To identify and quantify concentration in specific plant species under investigation, accurate analytical methods with broad applicability were developed and validated in the current study. Among various mobile phases tried, the mobile phase consisting of chloroform/methanol $(97: 3 \mathrm{~V} / \mathrm{V})$ demonstrated the best resolution between other peaks of the extract $[13,14]$.

The procedure for separation and the determination of various biomarkers in the methanolic fraction of C. longa using HPTLC-densitometry is reported at the six-point calibration curve in which curcumin was quantified with

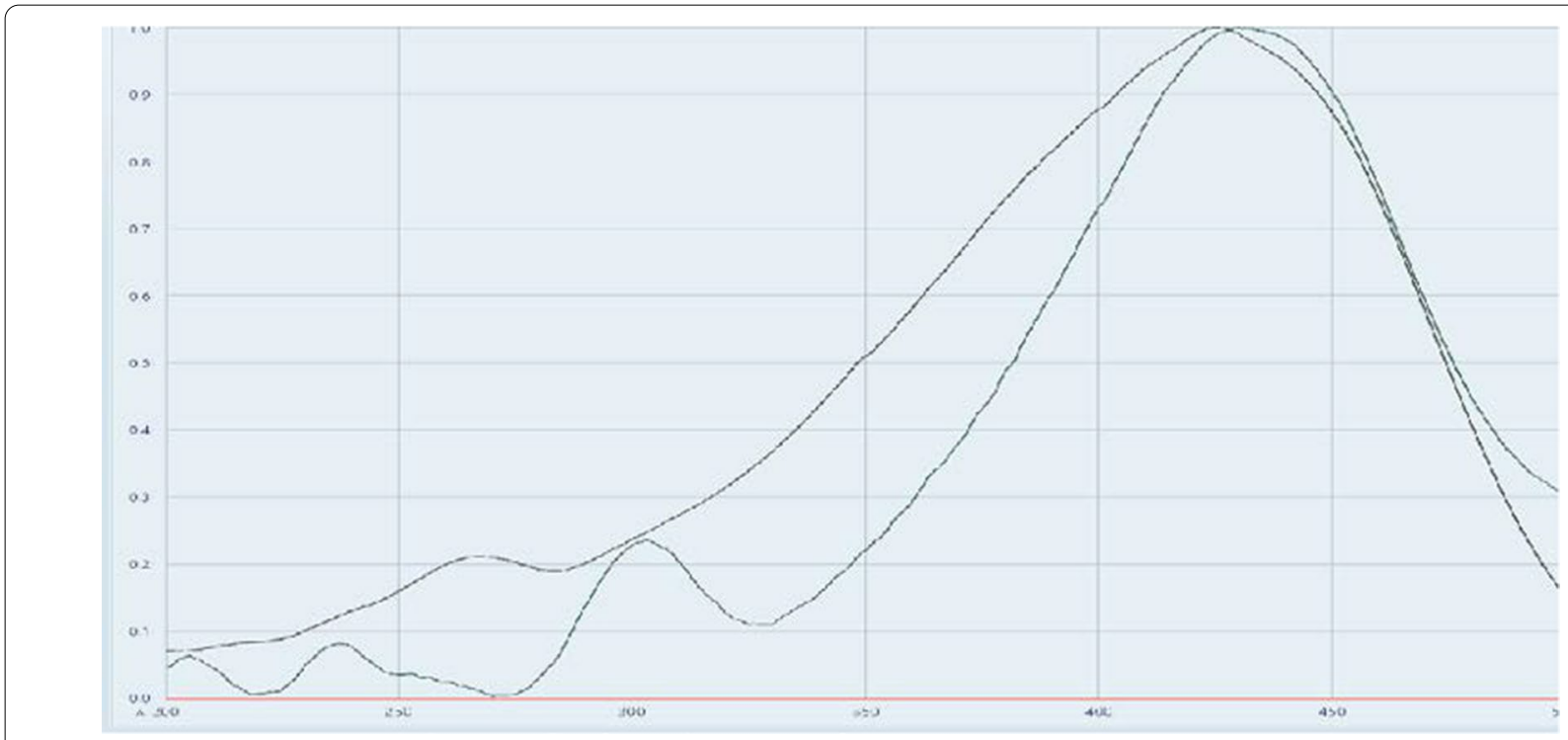

Fig. 5 HPTLC chromatograms of C. longa methanolic fraction with Curcumin 
Table 4 Quantification of curcumin in the methanolic fraction of C. longa

\begin{tabular}{ll}
\hline Plant sample & Curcumin (\%) \\
\hline C. longa & 1.5 \\
\hline
\end{tabular}

the method validation. The validation of the method was based on criteria of linearity, repeatability, selectivity, LOD, LOQ, robustness, and recovery. Inter-day analysis showed SD are within the limits of the $\mathrm{ICH}$ guidelines and indicates that the method is precise and reproducible for quantification of targeted metabolites under the developed protocol. The recovery of curcumin in $C$. longa extract showed good recovery. Results of the recovery studies indicate the high accuracy of the method. The modifications in the chromatographic conditions did not affect the detection of curcumin content $[6,14,18]$.

\section{Conclusions}

A simple, rapid, precise, accurate, and sensitive HPTLC method was successfully developed and validated for the determination of curcumin in C. longa. The developed method was found suitable for rapid screening of curcumin for their quantitative assessment. It does not necessitate any complex treatment or sophisticated analytical units, which are usually associated with HPLC and GC analyses. The obtained data suggest that the method is selective, reproducible, and economical and could be used for the routine analysis of reported compounds in crude drugs and extracts. The developed HPTLC method will help in the determination of the purity of the $C$. longa available from the different sources by detection of the related impurities as well as for quality control of C. longa. The HPTLC analysis has shown the presence of a quantifiable amount of curcumin in the samples. The content of the active principle, i.e., curcumin was found to be $1.5 \%$ (per dry weight). The developed method finds application in the pharmaceutical industry as a pharmacognostic tool for the quantification of this medicinally important plant.

\footnotetext{
Abbreviations

C. longa: Curcuma longa L; HPTLC: High-Performance Thin-Layer Chromatography; SMS-LLME: Supramolecular Solvent-Based Liquid-Liquid Microextraction; HPLC: High-Performance Liquid Chromatography (HPLC); UPLC-MS: Ultraperformance Liquid Chromatography-Mass Spectrometry (UPLC-MS); RPLC: Reversed-Phase Liquid Chromatography; ICH: International Conference on Harmonization; LOD: Limits of Detection (LOD); LOQ: Limits of Quantification; RSD: Relative Standard Deviation.
}

\section{Acknowledgements}

The authors are thankful to the Director, CSIR-NBRI and Faculty of Pharmacy, Integral University, Lucknow, for providing all the necessary facilities related to the present work (Manuscript Communication Number: IU/R\&D/2021- MCN0001139).

\section{Plant authentication:}

Identification and authentication of plant was carried by Dr. Syeda Khatoon, at CSIR-National Botanical Research Institute, Lucknow, and voucher specimens were submitted in LWG herbarium.

\section{Authors' contributions}

PK make substantial contribution to conception and design. BS and JD participated in the analysis and interpretation of data. BS, JD, and SS carried out acquisition of data and wrote the paper with input from all authors. All authors read and approved the final manuscript.

\section{Funding}

None.

Availability of data and materials

Data and material are available upon request.

\section{Declarations}

Ethics approval and consent to participate

Not applicable.

Consent for publication

Not applicable.

Competing interests

There is no conflict of interest.

\section{Author details}

${ }^{1}$ Faculty of Pharmacy, Integral University, Lucknow, India. ${ }^{2}$ Department of Pharmacy, Pranveer Singh Institute of Technology, Kanpur, India.

Received: 20 May 2021 Accepted: 22 August 2021

Published online: 28 August 2021

\section{References}

1. Sahu R, Saxena J (2013) Screening of total phenolic and flavonoid content in conventional and non-conventional species of curcuma. J Pharmacogn Phytochem 2:2278-4136

2. Kotha RR, Luthria DL (2019) Curcumin: biological, pharmaceutical, nutraceutical, and analytical aspects. Molecules 24(16):2930

3. Hewlings SJ, Kalman DS (2017) Curcumin: a review of its effects on human health. Foods 6(10):92

4. Al-Busaid MM, Akhtar MS, Alam T, Shehata WA (2020) Development and evaluation of herbal cream containing Curcumin from Curcuma longa. Pharm Pharmacol Int J 8(5):285-289

5. Kharat S, Namdeo AG, Mehta P (2017) Development and validation of HPTLC method for simultaneous estimation of curcumin and galangin in polyherbal capsule dosage form. J Taibah Univ Sci 11:775-781

6. Kadam PV, Yadav KN, Bhingare CL, Patil MJ (2018) Standardization and quantification of curcumin from Curcuma longa extract using UV visible spectroscopy and HPLC. J Pharmacogn Phytochem 7(5):1913-1918

7. Caleb J, Alshana U (2021) Supramolecular solvent-liquid-liquid microextraction followed by smartphone digital image colorimetry for the determination of curcumin in food samples. Sustain Chem Phar 21:100424

8. Setyaningsih D, Santoso YA, Hartini YS, Murti YB, Hinrichs WLJ, Patramurti C (2021) Isocratic high-performance liquid chromatography (HPLC) for simultaneous quantification of curcumin and piperine in a microparticle formulation containing Curcuma longa and Piper nigrum. Heliyon 7(3): e06541

9. Khorshidi N, Rahimi M, Salimikia I (2021) Application of aeration-assisted homogeneous liquid-liquid microextraction procedure using BoxBehnken design for determination of curcumin by HPLC (2020) J Sep Sci 43(13):2513-2520 
10. Sandhiutami NMD, Arozal W, Louisa M, Rahmat D (2021) Determine curcumin concentration in organ rats and in ovaries at ovarian cancer model rats using Ultra Performance Liquid Chromatography-Mass Spectrometry (UPLC-MS). Pharm Sci Asia 48(1):37-45

11. Rodriguez EL, Zhang C, Woolfork AG, Li Z, Bi C, Kaur H, Juritsch AF, Moreau R, Hage DS (2021) Analysis of curcumin and piperine in biological samples by reversed-phase liquid chromatography with multi-wavelength detection. J Chromatogr B; 1162:122487

12. Dwivedi J, Gupta A, Verma S, Paliwal S, Rawat AK (2019) Validated simultaneous high-performance thin layer chromatographic analysis of ursolic acid, $\beta$-sitosterol, lupeol and quercetin in the methanolic fraction of Ichnocarpus frutescens. J Planar Chromat 2(2):103-108

13. Dwivedi J, Gupta A, Verma S, Dwivedi M, Paliwal S, Rawat AK (2018) Validated high-performance thin-layer chromatographic analysis of ursolic acid and $\beta$-sitosterol in the methanolic fraction of Paederia foetida L. leaves. J Planar Chromat 31(5):377-381

14. Dwivedi J, Gupta A, Paliwal S, Rawat AK (2020) Validated simultaneous HPTLC analysis of scopoletin and gallic acid in the methanolic fraction of Jatropha glandulifera. J Planar Chromat 33(5):457-462

15. Sibug SM, Padolina ID, Cruz P, Garcia FC, Garrovillas MJ, Yabillo MR, Enriquez EP (2019) Smartphone-based image analysis and chemometric pattern recognition of the thin-layer chromatographic fingerprints of herbal materials. Anal Methods 11(6):721-732

16. Tosato F, Rosa TR, Morais CLM, Maldaner AO, Ortiz RS, Filgueiras PR, Lima KMG, Romão W (2016) Direct quantitative analysis of cocaine by thinlayer chromatography plus a mobile phone and multivariate calibration: a cost-effective and rapid method. Anal Methods 8:7632-7637

17. ICH-Q2A (1994) Text on validation of analytical procedures. In: Harmonized tripartite guideline prepared within the international conference on harmonization of technical requirements for the registration of pharmaceuticals for human use, Geneva

18. ICH-Q2B (1996) Validation of analytical procedures: methodology. In: Harmonized tripartite guideline prepared within the international conference on harmonization of technical requirements for the registration of pharmaceuticals for human use, Geneva

\section{Publisher's Note}

Springer Nature remains neutral with regard to jurisdictional claims in published maps and institutional affiliations.

\section{Submit your manuscript to a SpringerOpen ${ }^{\circ}$ journal and benefit from:}

- Convenient online submission

- Rigorous peer review

- Open access: articles freely available online

- High visibility within the field

- Retaining the copyright to your article

Submit your next manuscript at $\boldsymbol{\nabla}$ springeropen.com 